\title{
Disentangling Rotational Dynamics and Ordering Transitions in a System of Self- Organizing Protein Nanorods via Rotationally Invariant Latent Representations \\ Supplementary Material
}

\author{
Sergei V. Kalinin,, ${ }^{1,}$ Shuai Zhang, ${ }^{2,3}$ Mani Valleti, ${ }^{4}$ \\ Harley Pyles, ${ }^{5,6}$ David Baker, ${ }^{5,6,7}$ James J. De Yoreo, ${ }^{2,3}$ and Maxim Ziatdinov ${ }^{1,8, \dagger}$
}

${ }^{1}$ Center for Nanophase Materials Sciences, Oak Ridge National Laboratory, Oak Ridge, TN 37831, USA

${ }^{2}$ Materials Science and Engineering, University of Washington, Seattle, WA 98195, USA

${ }^{3}$ Physical Sciences Division, Pacific Northwest National Laboratory, Richland, WA 99354, USA

${ }^{4}$ Bredesen Center for Interdisciplinary Research, University of Tennessee, Knoxville, TN 37996, USA

${ }^{5}$ Department of Biochemistry, University of Washington, Seattle, WA 98195, USA

${ }^{6}$ Institute for Protein Design, University of Washington, Seattle, WA 98195, USA

${ }^{7}$ Howard Hughes Medical Institute, University of Washington, Seattle, WA 98195, USA

${ }^{8}$ Computational Sciences and Engineering Division, Oak Ridge National Laboratory, Oak Ridge, TN 37831, USA

*sergei2@ornl.gov

+ ziatdinovma@ornl.gov 


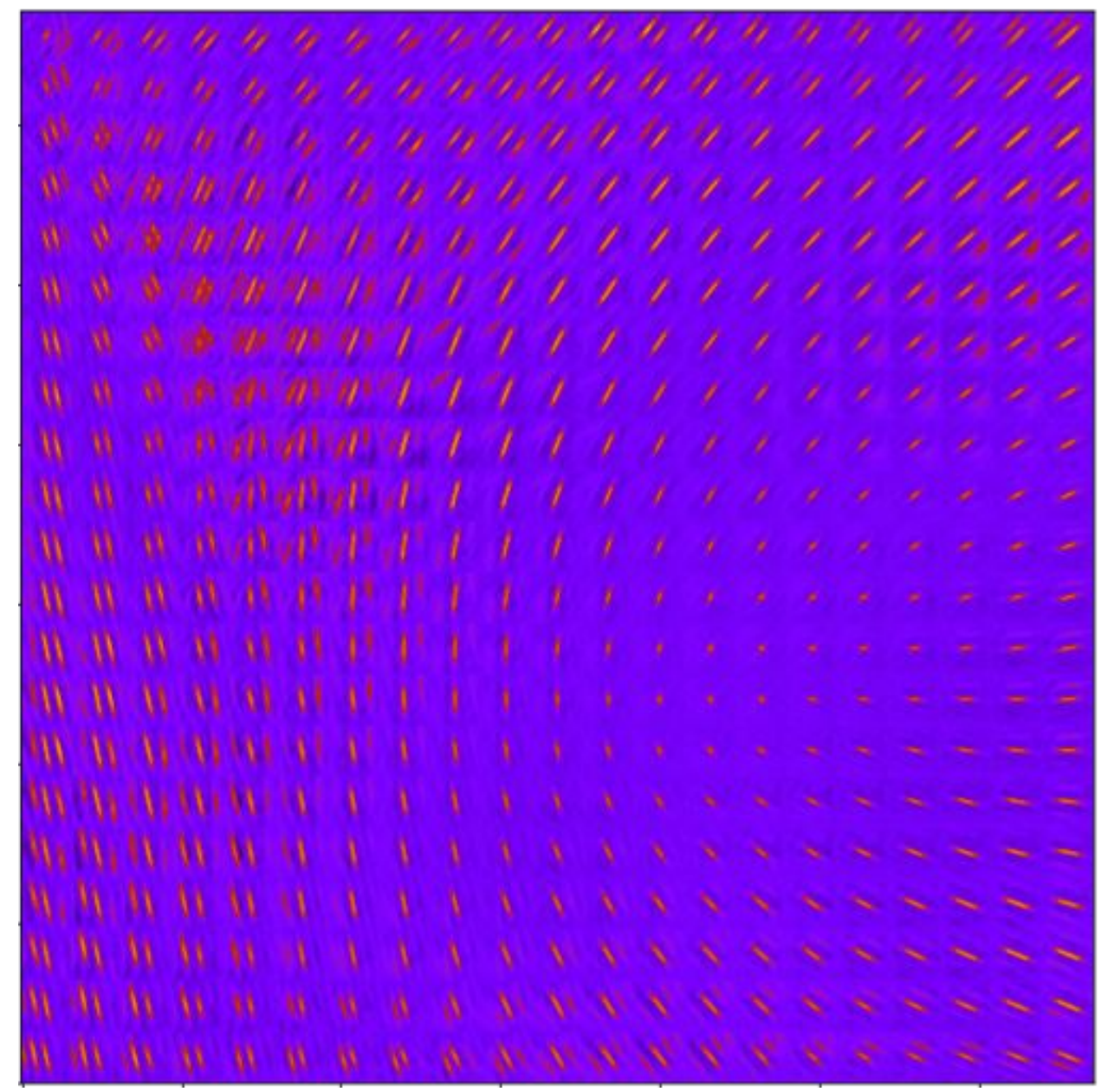

Supplementary Figure 1. The learned latent manifold of a regular VAE model projected onto the image space. 